\title{
The Tie2 signaling pathway in retinal vascular diseases: a novel therapeutic target in the eye
}

\author{
Quan Dong Nguyen ${ }^{1 *} \mathbb{C}$, Jeffrey S. Heier ${ }^{2}$, Diana V. Do ${ }^{1}$, Adam C. Mirando $^{3}$, Niranjan B. Pandey $^{3}$, Huan Sheng ${ }^{3}$
} and Theresa Heah ${ }^{3}$

\begin{abstract}
Background: Retinal vascular diseases such as neovascular age-related macular degeneration, diabetic retinopathy and/or diabetic macular edema, and retinal vein occlusion with macular edema-share several key pathophysiologic aspects including neovascularization, vascular permeability, and inflammation. The role of vascular endothelial growth factor (VEGF) in these processes, and the therapeutic benefits of VEGF inhibition, have been well characterized. AntiVEGF therapy is highly effective for many patients but is not uniformly effective in all patients and imposes a significant treatment burden. More recently, the role of the Tie2 signaling pathway in the pathophysiology of retinal vascular diseases has been investigated, and the Tie2 pathway represents a novel therapeutic target for these conditions.
\end{abstract}

Areas covered: The index review describes the Tie2 pathway and its complementary role to the VEGF pathway in the angiogenesis cascade and will summarize studies of molecules in development to therapeutically modulate the Tie2 pathway in retinal vascular diseases.

Conclusions: Activation of the Tie2 pathway leads to downstream signaling that promotes vascular health and stability and decreases vascular permeability and inflammation. AXT107 is a collagen IV-derived synthetic peptide with a dual mechanism of action that involves suppression of VEGF signaling and activation of the Tie2 pathway; these actions are accomplished by AXT107 binding to and disrupting different integrin, leading to blockade of the VEGF receptor and rearrangement of cellular Tie2 rendering it susceptible to Ang2 agonism. Other Tie2 agonist compounds are also in development, including faricimab and razuprotafib. Tie2 activation only modestly impacts angiogenesis on its own but significantly potentiates VEGF suppression. Co-regulation of the VEGF and Tie2 signaling pathways has the potential to improve functional and structural outcomes in eyes with retinal vascular diseases.

Keywords: Tie2, Integrin, Vascular endothelial grwoth factor, Vascular permeability, AXT107

\section{Background}

\section{Retinal vascular disease in the anti-VEGF Era}

The tissues of the eye in general, and the retina more specifically, are highly metabolically active and thus richly vascularized. Consequently, the retina is subject to both

\footnotetext{
*Correspondence: ndquan@stanford.edu

1 Spencer Center for Vision Research, Byers Eye Institute, Stanford University, 2370 Watson Court, Suite 200, Palo Alto, CA 94303, USA

Full list of author information is available at the end of the article
}

primary and secondary vascular diseases. Common retinal vascular disorders include neovascular age-related macular degeneration (nAMD), diabetic retinopathy (DR) and/or diabetic macular edema (DME), and retinal vein occlusion (RVO). While these various entities have distinct, complex, and multifactorial pathogeneses and risk factors, they share several key aspects of pathophysiology, specifically uncontrolled neovascularization, increased vascular permeability, and unregulated inflammation.

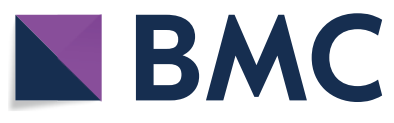

(c) The Author(s) 2020. This article is licensed under a Creative Commons Attribution 4.0 International License, which permits use, sharing, adaptation, distribution and reproduction in any medium or format, as long as you give appropriate credit to the original author(s) and the source, provide a link to the Creative Commons licence, and indicate if changes were made. The images or other third party material in this article are included in the article's Creative Commons licence, unless indicated otherwise in a credit line to the material. If material is not included in the article's Creative Commons licence and your intended use is not permitted by statutory regulation or exceeds the permitted use, you will need to obtain permission directly from the copyright holder. To view a copy of this licence, visit http://creativeco mmons.org/licenses/by/4.0/. The Creative Commons Public Domain Dedication waiver (http://creativecommons.org/publicdomain/ zero/1.0/) applies to the data made available in this article, unless otherwise stated in a credit line to the data. 
The development of therapies that inhibit vascular endothelial growth factor (VEGF) has revolutionized the management of retinal vascular diseases. In the presence of hypoxia, a state common to all ischemic retinal vascular diseases, VEGF synthesis is induced [1]. VEGF is a growth factor that plays a key role in angiogenesis and vascular permeability through its interaction with the VEGF receptor, a receptor tyrosine kinase [2]. VEGF levels are elevated in the aqueous and/or vitreous humor of eyes with nAMD, DR, DME, and RVO [3-5]. Multiple anti-VEGF therapies have been approved for the treatment of retinal vascular diseases-including ranibizumab, aflibercept, and brolucizumab-and another, bevacizumab, is often used off-label as well (Table 1) [6-9].

\section{Areas Covered}

The availability of anti-VEGF therapies has initiated a paradigm shift in the management of retinal vascular diseases. Yet, there remain unmet needs for the treatment of these conditions. The current pharmacologic agents are indicated for repeated intravitreal (IVT) injections every 1-3 months [6-8], although less often retreatment has shown to be effective [10-15]. This significant treatment burden is unsustainable, and many patients receive fewer than the recommended number of injections with commensurately suboptimal visual outcomes [16-21]. More importantly, a proportion of eyes fail to attain optimal visual outcomes even when receiving anti-VEGF therapy at recommended intervals [22, 23].

Table 1 Anti-VEGF drugs and their approved indications

\begin{tabular}{|c|c|}
\hline Drug & Approved indications \\
\hline Ranibizumab [6] & nAMD, DR, DME, RVO, myopic CNV \\
\hline Aflibercept [7] & nAMD, DR with DME, DME, RVO \\
\hline Brolucizumab [8] & nAMD \\
\hline Bevacizumab [9] & None (but often used for all of the above) \\
\hline
\end{tabular}

Such suboptimal outcome is likely because VEGF is only one component of the complex pathophysiology of retinal vascular diseases. Recent research has clarified the critical role of the Tie2 and other pathways-including those related to oxidative stress [24], the complement pathway [25], and others [26] - in the development of retinal vascular diseases including nAMD, DR/DME, and RVO [27-29]. There has been significant work to evaluate the Tie 2 pathway. The Tie2 signaling pathway, working in concert with the VEGF signaling pathway, represents a novel therapeutic target for these conditions. Several candidate drugs are in various stages of development, including AXT107, faricimab, and razuprotafib (Table 2). Among these, AXT107 is a collagen IV-derived peptide which acts as an inhibitor of vascular endothelial growth factor receptor 2 (VEGFR2) and an activator of the Tie2 pathway with potential therapeutic activity in nAMD, DME, RVO, and other retinal vascular diseases.

The index review will describe the Tie2 pathway and its role in the pathophysiology of retinal vascular diseases. Current drugs in development will also be reviewed, and the potential roles of these drugs that target the Tie2 pathway in the comprehensive management of retinal vascular diseases will be discussed.

\section{Overview of the Tie2 pathway}

Angiopoietin-1 (Ang1) and angiopoietin-2 (Ang2) are peptide ligands for the tyrosine kinase with Ig (immunoglobulin) and EGF (epidermal growth factor) homology domains 2 (Tie2) receptor [30]. Ang1 is a Tie2 receptor agonist while Ang2 is a contextual antagonist, blocking Tie2 signaling in most contexts but capable of enhancing Tie2 signaling under specific conditions (discussed below). The Tie 2 signaling pathway is specific to vascular endothelial cells [30-33] as is the VEGF pathway. However, while the VEGF pathway promotes the initiation of angiogenesis, the Tie 2 signaling pathway is more active in maintenance of vascular health, promoting endothelial cell (EC) survival, maturation, and stability [34].

Table 2 Investigational drugs targeting Tie2 pathway

\begin{tabular}{|c|c|c|c|c|}
\hline & AXT107 & Faricimab & Razuprotafib & Nesvacumab \\
\hline Sponsors & AsclepiX & Roche & Aerpio & Regeneron \\
\hline Structure & Peptide & Bispecific antibody & Small molecule & Monoclonal antibody \\
\hline Biological effect & $\begin{array}{l}\text { Inhibit VEGFR-A/-C } \\
\text { Activate Tie2 }\end{array}$ & Inhibit VEGFR-A and Ang-2 & Activate Tie2 & Inhibit Ang-2 \\
\hline Molecular weight (kDa) & 2.357 & 150 & 0.587 & 144.9 \\
\hline Route and Dose frequency & IVT every 6-12 months & IVT every 3-4 months & Subcutenous twice a day & IVT every 4 weeks \\
\hline Development status & $\begin{array}{l}\text { IND filing late } 2020 \\
\text { for DME, nAMD and } \\
\text { RVO }\end{array}$ & Phase 3 underway for nAMD and DME & Phase 2 for DME completed & Development terminated \\
\hline
\end{tabular}




\section{Key components of the Tie 2 pathway}

There are 5 key components of the Tie 2 pathway relevant to ocular angiogenesis: the Tie1 and Tie 2 receptors, angiopoietin-1 and -2 , and the vascular endothelial tyrosine phosphatase (VE-PTP) receptor.

Tie1 and Tie2 are related transmembrane receptor tyrosine kinases that share $76 \%$ homology in their intracellular domains and 33\% homology in their extracellular domains [35]. These receptors are expressed primarily on vascular endothelial cells (ECs), and can also be found on some hematopoietic cells. The extracellular portion of both receptors consist of Ig-like and fibronectin type III domains, and the intracellular portions are split tyrosine kinase domains. Tie2 binds both Ang1 and Ang2 at its extracellular Ig-like domains [35]; Tie1 does not bind the angiopoietins and its functional role in Ang1/Ang2 signaling is unclear [36].

Ang1 and Ang2 are Tie2 receptor ligands; both bind to Tie2 at the same site with similar affinity [37]. Ang1 is a Tie2 receptor agonist expressed by smooth muscle cells, pericytes and fibroblasts [33, 38]. Ang2 is a Tie2 receptor antagonist in resting ECs $[39,40]$, although it may also serve as a partial agonist when ECs are in activated or stressed states [41] such as in the setting of inflammation [42]. Ang2 is expressed primarily in ECs [38]. While both Ang1 and Ang2 form clusters, the Ang1 clusters contain greater number of molecules. The greater number of Ang1 molecules results in more extensive aggregation of Tie2 than what Ang2 can achieve, which is important for the strong activation of Tie2 [43-45].

VE-PTP is a transmembrane receptor tyrosine phosphatase that dephosphorylates Tie2 and downregulates Tie2 signaling [46, 47]. Inhibition of VE-PTP promotes Tie 2 activation by maintaining Tie2 in the active, phosphorylated state [48].

\section{Functions of the Tie2 pathway}

Ang1 binding to the Tie2 receptor activates Tie2 signaling (Fig. 1). The process begins with clustering and phosphorylation of the Tie2 receptor, which activates several downstream signaling pathways that support EC health. Akt (or protein kinase B) activation promotes EC survival and preservation $[49,50]$, suppresses Ang2 production via inactivation of transcription factor FOXO1 [51] and promotes vascular stability in EC $[52,53]$. The phosphatidylinositol 3-kinase (PI3K) pathway is also activated, promoting EC migration [54]. NF- $\mathrm{KB}$ mediated inflammation is suppressed by Tie2 activation [55]. EC permeability is also decreased, and overall EC health is supported and maintained through other incompletely characterized signaling pathways [54]. Ang2 binding disrupts the Tie2 clusters formed by Ang1 and blocks its pro-vascular stability effects. Consequences include abnormal vascular structure formation [56], increased vascular permeability [56], and increased inflammation [57].

Animal studies have demonstrated these effects well. Tie2 deficient mice are non-viable in the second week of gestation; a rudimentary vascular system appears but fails to remodel or mature, with few branches, pericytes, or smooth muscle cells [58, 59]. Ang1-deficient mice share this phenotype with Tie2-deficient mice [60], while overexpression of Ang1 in skin cells produces hypervascularity (vessels are more numerous, larger, and more highly branched) [61] with decreased vascular permeability [62]. Ang2-deficient mice survive but have altered vascularization (abnormal retinal vascularization, failure of regression of hyaloid vessels of the crystalline lens) [63], while overexpression of Ang2 disrupts vascular formation leading to mid-gestational death similar to Ang1 deficiency [32].

\section{Role of the Tie2 pathway in retinal vascular diseases}

The various retinal vascular diseases are characterized by posterior segment neovascularization and/or vasculopathy followed by fluid extravasation into tissues from these permeable new or impaired vessels and are frequently associated with inflammation. Several lines of research demonstrate a pathophysiological role for the Ang-Tie pathway in these conditions.

\section{Neovascularization}

Choroidal neovascularization is a hallmark of nAMD. Ang2 is a susceptibility gene for the development of nAMD [64], and Ang2 levels are elevated in aqueous humor of eyes with nAMD and correlate with severity of disease [65]. Both Ang1 and Ang2, as well as VEGF, are expressed on excised subfoveal membranes from eyes with nAMD [66]; so is Tie2, and the highest levels of Ang2 and VEGF are from the most highly vascularized regions of these CNV membranes [67]. Preretinal and retinal neovascularization are hallmarks of PDR and $\mathrm{RVO}$, and vitreous levels of Ang2 are elevated in eyes with PDR [68] and RVO [69].

These findings illustrate that ocular neovascularization is driven in part by Tie 2 receptor antagonism by Ang2. Conversely, Ang1 suppresses choroidal neovascularization $(\mathrm{CNV})$ and vascular leakage in a laser-induced mouse model [70], demonstrating the potential therapeutic effects of targeting the Tie2 pathway to block the neovascularization process.

\section{Vascular permeability}

Leakage of fluid into and under the retina is a hallmark of many retinal vascular diseases. Vascular permeability is increased in these eyes through the combined activity 


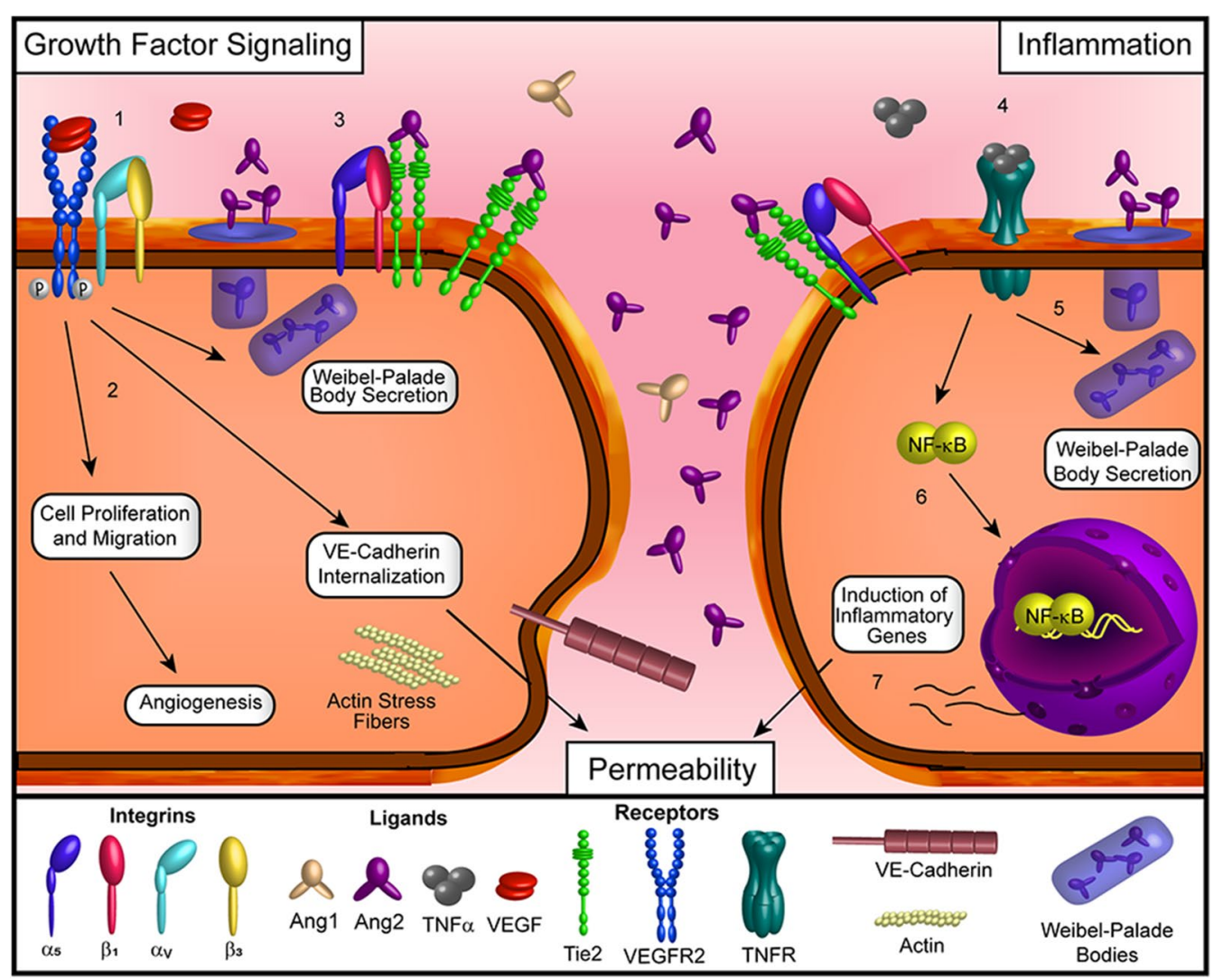

Fig. 1 VEGF and TNF signaling in retinal vascular disease. Increased expression of angiogenic and inflammatory factors (such as VEGF and TNFa) are associated with retinal vascular diseases. (1) The binding of VEGF to VEGFR2 in complex with $a_{v} \beta_{3}$ integrin stimulates the autophosphorylation of VEGFR2 and the activation of downstream cellular processes. (2) These processes include the induction of migration and proliferation important for angiogenesis and the reorganization of diffuse actin into stress fibers and the internalization of cell-cell adhesion proteins that result in increased permeability. (3) VEGFR2 signaling also stimulates the release of Ang2 from Weibel-Palade bodies, which then competes with Ang1 to bind to Tie2 and reduce the associated signaling pathways related to vascular stability and anti-permeability. (4) With respect to inflammatory signaling, the binding of TNFa to the TNFR also stimulates the release of Ang2 from Weibel-Palade bodies (5) and reduces the activation of Tie2. This not only reduces the vessel stabilizing pathways mentioned above but also blocks the anti-inflammatory activity of the Tie2 receptor as well. (6) The activation of the TNFR pathway by TNFa also stimulates the relocation of NF-KB to the nucleus where it functions as a transcription factor to induce the expression of numerous inflammation-associated genes. (7) Among other functions, the initiation of inflammation stimulates increased permeability within blood vessels

of the VEGF and Tie2 pathways. Ang2 potentiates the action of VEGF; VEGF-induced vascular permeability triples in the presence of Ang2 [71]. Ang2 is upregulated in settings of hypoxia [72], hyperglycemia [73, 74] and oxidative stress [75], which are common in eyes of diabetic patients, and Ang2 levels are elevated in the vitreous of diabetic eyes [76, 77], particularly those with retinopathy [78]. Ang2 is increased in sepsis [79, 80]-in which increased vascular permeability is a crucial componentand Ang2 levels correlate with the severity of sepsis [80]. In contrast, Ang1 functions as an anti-permeability growth factor and promotes EC remodeling to minimize permeability [81]. Likewise, Tie2 signaling decreases vascular permeability by tightening intra-EC junctions; this effect is mediated by associations of Tie 2 receptors on adjacent cells and reorganization of key adhesion molecule vascular endothelial-cadherin (VE-cadherin) complexes [82]. As examples of its critical role in regulating vascular permeability in health and disease, Tie2 activation reduces pulmonary vascular leakage during cardiopulmonary bypass in rats [83], reduces cerebrovascular permeability and stroke size in mouse models [84], and reduced renal vascular edema and transcapillary albumin flux is a mouse model of acute kidney injury [85].

\section{Inflammation}

Increased expression of pro-inflammatory cytokines in the retina has been observed in retinal vascular diseases [86-88]. TNF $\alpha$ is a primary cytokine involved in inflammatory disease of the eye. Ang2 can potentiate 
the activities of TNF $\alpha$. Under inflammatory conditions, Ang2 is rapidly secreted from EC and antagonizes the vessel-stabilizing activities of the Tie2 receptor by blocking its interaction with Ang1 [89].

The pathophysiology of macular edema, and its adverse effect on visual acuity, is driven by both the VEGF and Tie2 pathways, as well as synergistic interplay between the two. Ang1 blocks VEGF-induced vascular permeability without inhibiting VEGF-induced angiogenesis signalling [90]. The mechanism for the crosstalk responsible for regulating vascular permeability involves the sequestration of cell adhesion molecule VE-cadherin [91], which under normal conditions is located at cell junctions and maintains them but in the presence of VEGF is internalized in intracellular vesicles resulting in cell junction breakdown and increased permeability. Ang1 blocks VEGF-induced redistribution of VE-cadherin, thus preserving EC junctions and preventing increased permeability, through a series of complex interactions with Src-family kinases, Rho-family GTPases, and the formin $\mathrm{mDia}$ [90]. This crosstalk between the two pathways can be exploited therapeutically, as Tie2 activation suppresses VEGF-induced vascular leakage and reduces inflammation in mouse models $[92,93]$.

\section{Targeting the Tie2 pathway for retinal vascular diseases AXT107}

Angiogenesis is a complex multi-step process that requires remodeling of the ECM to make way for vascular growth and to support new vessels. The ECM plays a key regulatory role in all aspects of angiogenesis, including structural support for cells as well as molecular signaling for vascular sprouting, lumen formation, and vessel maturation and stability [94]. ECM degradation liberates numerous small peptides-including angiostatin, endostatin, and several small peptides derived from type IV collagen-that have anti-angiogenic properties and serve as natural regulators of the angiogenesis process $[95,96]$.

Numerous peptides based on ECM proteolysis products have been synthesized and screened in vitro for anti-angiogenic activity using a computational-based peptidomics approach [97]. Of these, several peptides derived from type IV collagen such as cyrostatin, the wispostatin family, and the chemokinostatin family have potent anti-angiogenic activity [97].

AXT107 (Table 2) is a 20-mer synthetic peptide derived from the non-collagenous domain of collagen IV that may be a triple threat to the pathophysiology of retinal vascular diseases: suppressing ocular neovascularization, inhibiting vascular leakage, and reducing inflammation [79, 92]. AXT107 targets two pathways in a single molecule: it promotes Tie2 pathway signaling [82] while blocking VEGF pathway signaling [92]. Both of these actions are mediated by AXT107's interaction with and disruption of the proteins integrin $\alpha_{v} \beta_{3}[82,92]$ and integrin $\alpha_{5} \beta_{1}[82,92,98-100]$.

Integrins are essential for the attachment of cells to the ECM. Integrin $\alpha_{5} \beta_{1}$ interacts with Tie 2 receptors and sequesters them at EC-ECM interface [82]. In this resting state, Ang2 is a Tie2 antagonist. In the presence of AXT107, however, integrin $\alpha_{5} \beta_{1}$ is disrupted and Tie2 receptor molecules can then reorganize within cells, clustering at EC-EC junctions. These clusters are presumed to be similar to the Tie 2 superclusters that form naturally in response to Ang1 exposure but are inactive in absence of a bound ligand. However, by precluding the requirement of the ligand to form these structures, either Ang1 or Ang 2 are able to bind and activate Tie2 signaling. The reorganization and junctional clustering of Tie2 induced by AXT107 allows Ang2, which normally functions as an antagonist of Tie2 and is an inducer of vascular leakage, to function as a strong agonist of Tie 2 and a promoter of vascular integrity. Tie2 phosphorylation then activates the myriad downstream signaling pathways that promote vascular integrity and health $[82,92]$.

AXT107 suppresses TNF $\alpha$-induced vascular inflammation in endothelial cells by converting the pro-inflammatory Ang2 into a strong agonist of Tie2 signaling by promoting junctional clustering of Tie2 as explained above, disrupting the synergism between TNF $\alpha$ and Ang2 while also preventing I $\mathrm{I} B \alpha$ degradation directly through Tie2 signaling (Fig. 2). The recovery of IkB $\alpha$ prevents NF- $\mathrm{kB}$ nuclear localization, thereby blocking NF- $\mathrm{kB}$ induced inflammatory responses $[55,93]$.

Integrin $\alpha_{\mathrm{v}} \beta_{3}$ also complexes with VEGF receptor 2 (VEGFR2) to enhance its downstream signaling when ligand-bound by VEGF [101]. In the presence of AXT107, integrin $\alpha_{v} \beta_{3}$ is disrupted and not available to complex with VEGFR2, so that VEGF-bound VEGFR2 phosphorylation is reduced, blocking downstream signaling [92]. In addition, VEGFR2 surface levels are reduced by degradation also contributing to reduced levels of signaling. Consequently, choroidal neovascularization and vascular leakage are inhibited.

AXT107 solution is delivered by intravitreal (IVT) injection in preclinical rabbit and minipig models. In the vitreous AXT107 self-assembles into a small gellike depot post-IVT injection and settles into the inferior vitreous, away from the visual axis, and dissipates over months [92]. In preclinical studies, AXT107 has been shown to suppress and regress $\mathrm{CNV}$ in a laserinduced mouse model [92], suppress subretinal neovascularization in rho/VEGF transgenic mice [92], suppress ischemia-induced retinal neovascularization in a mouse model of oxygen-induced ischemic 


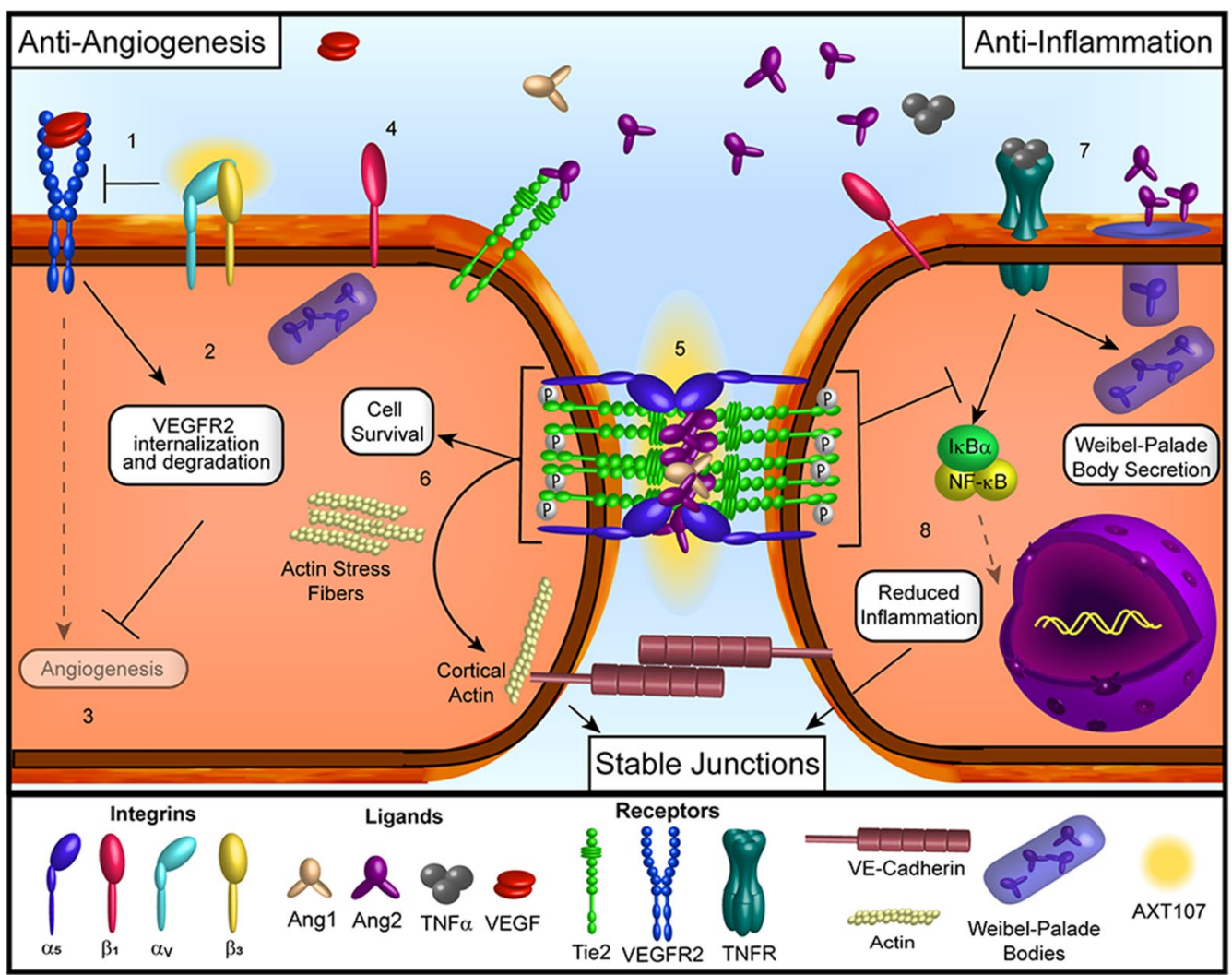

Fig. 2 Effects of AXT107-treatment in retinal vascular disease. (1) AXT107 binds to $a_{y} \beta_{3}$ integrins and dissociates them from VEGFR2. (2) The disruption of these interactions directly inhibits the autophosphorylation of VEGFR2 in the presence of VEGF and further reduces VEGFR2 responses by increasing the internalization and degradation of the receptor, overall reducing the angiogenesis responses and induction of vessel permeability (3). (4) AXT107 also binds to $a_{5} \beta_{1}$ integrin heterodimers, which associate with Tie2, and dissociates the subunits. (5) $a_{5}$ integrin and Tie2 then relocate to the endothelial cell-cell junctions and form clusters that can be activated following the binding of either Ang1 or Ang2. (6) Active Tie2 clusters stimulate downstream pathways associated with improved endothelial cell survival and the reorganization of intracellular actin from stress fibers into cortical actin that is distributed around the edges of the cell to stabilize the vasculature. (7) Initial activation of TNFR by TNFa does not seem to be affected by AXT107 treatment, resulting in the release of Ang2 from Weibel-Palade bodies. The released Ang2 contributes to the activation of Tie2 clusters formed following AXT107 treatment. (8) Signaling by the phosphorylated Tie2 clusters prevent the degradation of IKBa molecules, retaining NF-KB within the cytoplasm and blocking the induction of inflammation and the associated effects on vessel permeability, thereby contributing to increased vessel stability

retinopathy [92], suppress VEGF-induced vascular leakage in rho/VEGF transgenic mice and in Dutch Belted rabbits [92], and reduce inflammation in mouse model of TNF $\alpha$-induced inflammation and leukostasis [93]. In Dutch Belted rabbits, anti-permeability activity was still present after 60 days when re-challenged with VEGF in AXT107-treated eyes but not in aflibercepttreated eyes [92]. In an exploratory study with a single IVT injection of AXT107 in a modified Dutch Belted rabbit model of VEGF-induced vascular leakage, the AXT107 demonstrated inhibition of vascular leakage compared to VEGF controls throughout the 12-month duration. In addition, an ongoing PK study in Dutch Belted rabbit demonstrated drug level in the retina for at least 12 months (data on file). Such observations in rabbit eyes-which more closely approximate the size of human eyes than do mouse eyes-suggest the possibility of infrequent dosing of AXT107 in human eyes, which could significantly reduce the treatment burden posed by every 1-3 month retreatment of currently approved anti-VEGF agents for retinal vascular disease.

Clinical development of AXT107 will be initiated in late 2020 with the Investigational New Drug filing of phase $1 / 2$ a clinical studies to evaluate the safety and bioactivity of AXT107 in the management of DME, nAMD, and RVO.

Several other compounds that target the Tie2 signaling pathway are in various stages of clinical development (Table 2). 


\section{Faricimab}

Faricimab is a bispecific antibody that binds and blocks both VEGF and Ang2 without any binding to Ang1 $[58,102,103]$. The phase 2 AVENUE trial in eyes with treatment-naïve nAMD showed that faricimab given by IVT injection every 4 weeks (q4wk) provided better best-corrected visual acuity (BCVA) gains than ranibizumab q4wk or the combination of faricimab and ranibizumab each given q4wk at 36 weeks [104]. The phase 2 STAIRWAY trial in eyes with treatment-naïve nAMD showed that faricimab every 12 weeks (q12wk) and every 16 weeks (q16wk) provided comparable BCVA and anatomic outcomes [macular thickness on optical coherence tomography (OCT) imaging] after 1 year of therapy [105]. The phase 2 BOULEVARD trial in DME compared two doses of faricimab to monthly ranibizumab and found that the higher dose faricimab produced BCVA gains that were superior to ranibizumab at 24 weeks with no safety issues identified [106]. Phase 3 trials are underway for nAMD $[107,108]$ and DME $[109,110]$.

\section{Razuprotafib (formerly AKB-9778)}

Razuprotafib is a small molecule inhibitor of VE-PTP. Its inhibition leads to activation of Tie2 pathways and promotes all downstream signaling (thus stabilizing ocular vasculature) by promoting Tie2 phosphorylation, enhancing Ang2-induced Tie2 phosphorylation, and increasing Tie2 phosphorylation in the presence of Ang2 [111]. The drug is dosed by subcutaneous injection twice daily. A phase 1 trial in DME revealed that doses of $15 \mathrm{mg}$ or more resulted in improved BCVA and reductions in OCT central subfield thickness (CST) in some eyes with no systemic safety issues identified [112]. A phase 2 trial in DME compared razuprotafib in combination with ranibizumab to each component as monotherapy [113]. Improvements in BCVA were comparable with ranibizumab and the combination but minimal in the razuprotafib monotherapy group; greater CST reduction was seen in the combo group than either monotherapy group. These results suggested that Tie2 activation with VEGF inhibition reduces DME more than VEGF inhibition alone. No safety issues were identified. Two phase 2 trials were conducted in eyes with nonproliferative DR. In the first (TIME-2a), comparable proportions of eyes manifested $a \geq 2$-step improvement in DR scores in the ranibizumab, razuprotafib, and combination groups $(8.8 \%, 10.0 \%$, and $11.4 \%$, respectively) [114]. In the second (TIME-2b), there was no significant difference between razuprotafib and placebo in the proportions of eyes attaining $a \geq 2$-step improvement in DR scores [115]. This finding suggested that Tie2 activation requires concomitant VEGF suppression for optimal efficacy. A phase 2 study in eyes with macular edema secondary to
RVO was completed but not reported [116]. ARP-1536 is a humanized monoclonal antibody targeting VE-PTP with comparable efficacy to AKB-9778 but with intravitreal administration that is in development [117].

\section{Nesvacumab}

Nevascumab is a fully human IgG monoclonal antiAng2 antibody that binds Ang2 and blocks its binding to Tie2. It was co-formulated with aflibercept for intravitreal injection, and was well tolerated in a phase 1 trial in nAMD and DME with promising effects on BCVA [118] but failed to demonstrate superiority with respect to visual acuity gains in phase 2 studies in nAMD (ONYX) [119] and DME (RUBY) [120] and development was abandoned without phase 3 evaluation [121]. Despite the failure to demonstrate superiority in visual acuity, there were signals of anatomic and durability benefits.

\section{Summary and Conclusion}

Retinal vascular diseases such as nAMD, DME, and macular edema associated with RVO are characterized by the presence of neovascularization and/or increased vascular permeability, and the contribution from inflammatory processes. The VEGF signaling pathway generally regulates angiogenesis while the Tie 2 signaling pathway oversees the maturation and stability of the vasculature. Tie2 is a receptor tyrosine kinase with two key peptide ligands: angiopoietin-1 (Ang1) is a receptor agonist while angiopoietin-2 (Ang2) is primarily a receptor antagonist but can function as an agonist in some circumstances. Activation of the Tie2 pathway leads to downstream signaling that promotes vascular health and stability and decreases vascular permeability and inflammation.

The Tie2 pathway is active in ocular vascular beds, is integral to neovascularization and permeabiity in retinal vascular diseases, and represents a novel therapeutic target for these conditions. AXT107 is a collagen IV-derived synthetic peptide with a dual mechanism of action that involves suppression of VEGF signaling and activation of the Tie2 pathway; these actions are accomplished by AXT107 binding to and disrupting integrin $\alpha_{v} \beta_{3}$ and integrin $\alpha_{5} \beta_{1}$ leading to blockade of the VEGF receptor and rearrangement of cellular Tie2 rendering it susceptible to Ang2 agonism. In animal models, AXT107 suppresses neovascularization, decreases vascular permeability, and reduces inflammation. Other molecules in development to target the Tie2 pathway include faricimab, a bispecific antibody that blocks both VEGF and Ang2 (now in phase 3 trials for nAMD and DME) and razuprotafib, a small molecule inhibitor of the Tie2 inhibitor VE-PTP (effectively enhancing Tie 2 activity).

Tie2 activation only modestly impacts angiogenesis on its own but significantly potentiates VEGF suppression; 
co-regulation of the VEGF and Tie2 signaling pathways has the potential to improve functional and structural outcomes in eyes with retinal vascular diseases.

\section{Acknowledgements}

None.

\section{Authors' contributions}

Manuscript preparation: QDN, ACM, NBP, HS, TH. Critical review and approval for submission: QDN, JSH, DVD, ACM, NBP, HS, TH. All authors read and approved the final manuscript.

\section{Funding}

None

\section{Availability of data and materials}

Not applicable

\section{Ethics approval and consent to participate and publish}

Not applicable.

\section{Consent for publication}

Not applicable.

\section{Competing interests}

QDN serves on the Scientific Advisory Board for AbbVie, Bayer, Genentech, Mallinckrodt, Regeneron, and Santen, among others and advises on Drug Safety for AsclepiX; QDN also chaired the Steering Committee for the RIDE/ RISE and STOP-Uveitis Study and was on the Steering Committee for other studies sponsored by Genentech and Regeneron.JSH serves on the Scientific Advisory Board for 4DMT, Adverum, Aerie, Aerpio, Akros, Allegro, Apellis, Array, Asclepix, Bayer, Beaver-Visitec, BioMarin, Clearside, Corcept, Daiichi Sankyo, Galecto, Galimedix, Genentech/Roche, Helio, Hemera, Interface, iRenix, Janssen, Kanghong, Kodiak, Notal Vision, Novartis, Ocular Therapeutix, Optos, Orbit Biomedical, Quark, Ra Pharmaceuticals, Regeneron, REGENXBIO, Santen, Scifluor, Shire, Spark Therapeutics, Stealth, Thrombogenics, Tyrogenex.DVD serves on the Scientific Advisory Board for Allergan, AsclepiX, Genentech, Kodiak, Regeneron, and Santen and she has received research support from Genentech and Regeneron.ACM, NBP, HS, and TH are employees of AsclepiX Therapeutics.

\section{Author details}

1 Spencer Center for Vision Research, Byers Eye Institute, Stanford University, 2370 Watson Court, Suite 200, Palo Alto, CA 94303, USA. ${ }^{2}$ Ophthalmic Consultants of Boston, Boston, MA, USA. ${ }^{3}$ AsclepiX Therapeutics, Baltimore, MD, USA

Received: 6 June 2020 Accepted: 15 September 2020

Published online: 13 October 2020

\section{References}

1. Shweiki D, Itin A, Soffer D, Keshet E. Vascular endothelial growth factor induced by hypoxia may mediate hypoxia-initiated angiogenesis. Nature. 1992;359:843-5.

2. Ferrara N. Vegf and intraocular neovascularization: from discovery to therapy. TransI Vis Sci Technol. 2016;5:10.

3. Aiello LP, Avery RL, Arrigg PG, et al. Vascular endothelial growth factor in ocular fluid of patients with diabetic retinopathy and other retinal disorders. N Engl J Med. 1994;331:1480-7.

4. Funk M, Karl D, Georgopoulos M, et al. Neovascular age-related macular degeneration: intraocular cytokines and growth factors and the influence of therapy with ranibizumab. Ophthalmology. 2009;116:2393-9.

5. Funatsu H, Yamashita H, Sakata K, et al. Vitreous levels of vascular endothelial growth factor and intercellular adhesion molecule 1 are related to diabetic macular edema. Ophthalmology. 2005:112:806-16.

6. Genentech. Lucentis Prescribing Information. 2017.

7. Aflibercept Prescribing Information. https://www.accessdata.fda.gov/ drugsatfda_docs/label/2017/125387s054lbl.pdf. Accessed 15 April 2018
8. Novartis. Beovu Prescribing Information. 2019.

9. Low A, Faridi A, Bhavsar KV, et al. Comparative effectiveness and harms of intravitreal antivascular endothelial growth factor agents for three retinal conditions: a systematic review and meta-analysis. Br J Ophthalmol. 2019;103:442-51.

10. Wykoff CC, Ou WC, Brown DM, et al. Randomized trial of treat-andextend versus monthly dosing for neovascular age-related macular degeneration: 2-year results of the trex-amd study. Ophthalmol Retina. 2017:1:314-21.

11. Silva R, Berta A, Larsen M, et al. Treat-and-extend versus monthly regimen in neovascular age-related macular degeneration: results with ranibizumab from the trend study. Ophthalmology. 2018;125:57-65.

12. Kertes PJ, Galic IJ, Greve M, et al. Canadian treat-and-extend analysis trial with ranibizumab in patients with neovascular age-related macular disease: one-year results of the randomized canadian treat-and-extend analysis trial with ranibizumab study. Ophthalmology. 2019;126:841-8.

13. Berg K, Hadzalic E, Gjertsen I, et al. Ranibizumab or bevacizumab for neovascular age-related macular degeneration according to the lucentis compared to avastin study treat-and-extend protocol: two-year results. Ophthalmology. 2016;123:51-9.

14. DeCroos FC, Reed D, Adam MK, et al. Treat-and-extend therapy using aflibercept for neovascular age-related macular degeneration: a prospective clinical trial. Am J Ophthalmol. 2017;180:142-50.

15. Ohji $\mathrm{M}$, Takahashi K, Okada AA, et al. Efficacy and safety of intravitreal aflibercept treat-and-extend regimens in exudative age-related macular degeneration: 52- and 96-week findings from altair: a randomized controlled trial. Adv Ther. 2020:37:1173-87.

16. Gillies MC, Nguyen V, Daien V, Arnold JJ, Morlet N, Barthelmes D. Twelve-month outcomes of ranibizumab vs. aflibercept for neovascular age-related macular degeneration: data from an observational study. Ophthalmology. 2016:123:2545-53.

17. Holz FG, Bandello F, Gillies M, et al. Safety of ranibizumab in routine clinical practice: 1-year retrospective pooled analysis of four european neovascular amd registries within the luminous programme. $\mathrm{Br} J \mathrm{Oph}-$ thalmol. 2013:97:1161-7.

18. Holz FG, Tadayoni R, Beatty S, et al. Multi-country real-life experience of anti-vascular endothelial growth factor therapy for wet age-related macular degeneration. Br J Ophthalmol. 2015;99:220-6.

19. Writing Committee for the, U. K. Age-Related Macular Degeneration E. M. R. Users Group. The neovascular age-related macular degeneration database: multicenter study of 92976 ranibizumab injections: report 1: visual acuity. Ophthalmology. 2014:121:1092-101.

20. Kim LN, Mehta H, Barthelmes D, Nguyen V, Gillies MC. Metaanalysis of real-world outcomes of intravitreal ranibizumab for the treatment of neovascular age-related macular degeneration. Retina. 2016:36:1418-31.

21. Wells JA, Glassman AR, Ayala AR, et al. Aflibercept, bevacizumab, or ranibizumab for diabetic macular edema: two-year results from a comparative effectiveness randomized clinical trial. Ophthalmology. 2016;123:1351-9.

22. Broadhead GK, Hong T, Chang AA. Treating the untreatable patient: current options for the management of treatment-resistant neovascular age-related macular degeneration. Acta Ophthalmol. 2014;92:713-23.

23. Hussain RM, Ciulla TA. Treatment strategies for refractory diabetic macular edema: switching anti-vegf treatments, adopting corticosteroid-based treatments, and combination therapy. Expert Opin Biol Ther. 2016:16:365-74.

24. Abokyi S, To CH, Lam TT, Tse DY. Central role of oxidative stress in age-related macular degeneration: evidence from a review of the molecular mechanisms and animal models. Oxid Med Cell Longev. 2020;2020:7901270

25. Wu J, Sun X. Complement system and age-related macular degeneration: drugs and challenges. Drug Design Dev Therapy. 2019;13:2413-25.

26. Choudhary M, Malek G. A review of pathogenic drivers of age-related macular degeneration, beyond complement, with a focus on potential endpoints for testing therapeutic interventions in preclinical studies. Adv Exp Med Biol. 2019;1 185:9-13.

27. Hussain RM, Neiweem AE, Kansara V, Harris A, Ciulla TA. Tie-2/angiopoietin pathway modulation as a therapeutic strategy for retinal disease. Expert Opin Investig Drugs. 2019;28:861-9. 
28. Miller K, Fortun JA. Diabetic macular edema: current understanding, pharmacologic treatment options, and developing therapies. Asia Pac J Ophthalmol (Phila). 2018;7:28-35.

29. Campochiaro PA. Molecular pathogenesis of retinal and choroidal vascular diseases. Prog Retin Eye Res. 2015;49:67-81.

30. Partanen J, Armstrong E, Makela TP, et al. A novel endothelial cell surface receptor tyrosine kinase with extracellular epidermal growth factor homology domains. Mol Cell Biol. 1992;12:1698-707.

31. Sato TN, Qin Y, Kozak CA, Audus KL. Tie-1 and Tie-2 define another class of putative receptor tyrosine kinase genes expressed in early embryonic vascular system. Proc Natl Acad Sci USA. 1993;90:9355-8.

32. Maisonpierre PC, Suri C, Jones PF, et al. Angiopoietin-2, a natural antagonist for Tie2 that disrupts in vivo angiogenesis. Science. 1997;277:55-60

33. Davis $\mathrm{S}$, Aldrich $\mathrm{TH}$, Jones PF, et al. Isolation of angiopoietin-1, a ligand for the Tie2 receptor, by secretion-trap expression cloning. Cell. 1996;87:1161-9.

34. Thurston $\mathrm{G}$, Daly $\mathrm{C}$. The complex role of angiopoietin- 2 in the angiopoietin-tie signaling pathway. Cold Spring Harb Perspect Med. 2012;2:a006550.

35. Schnurch $\mathrm{H}$, Risau W. Expression of Tie-2, a member of a novel family of receptor tyrosine kinases, in the endothelial cell lineage. Development. 1993;119:957-68

36. Saharinen P, Kerkela K, Ekman N, et al. Multiple angiopoietin recombinant proteins activate the Tie1 receptor tyrosine kinase and promote its interaction with Tie2. J Cell Biol. 2005;169:239-43.

37. Fiedler U, KrissI T, Koidl S, et al. Angiopoietin-1 and angiopoietin-2 share the same binding domains in the Tie-2 receptor involving the first Ig-like loop and the epidermal growth factor-like repeats. J Biol Chem. 2003;278:1721-7

38. Stratmann A, Risau W, Plate KH. Cell type-specific expression of angiopoietin-1 and angiopoietin-2 suggests a role in glioblastoma angiogenesis. Am J Pathol. 1998;153:1459-66.

39. Reiss Y, Droste J, Heil M, et al. Angiopoietin-2 impairs revascularization after limb ischemia. Circ Res. 2007;101:88-96.

40. Scharpfenecker M, Fiedler U, Reiss Y, Augustin HG. The Tie-2 ligand angiopoietin-2 destabilizes quiescent endothelium through an internal autocrine loop mechanism. J Cell Sci. 2005:118:771-80.

41. Daly C, Pasnikowski E, Burova E, et al. Angiopoietin-2 functions as an autocrine protective factor in stressed endothelial cells. Proc Natl Acad Sci USA. 2006;103:15491-6.

42. Tabruyn SP, Colton K, Morisada T, et al. Angiopoietin-2-driven vascular remodeling in airway inflammation. Am J Pathol. 2010;177:3233-43.

43. Kim KT, Choi HH, Steinmetz MO, et al. Oligomerization and multimerization are critical for angiopoietin-1 to bind and phosphorylate Tie2. J Biol Chem. 2005;280:20126-31.

44. Moore JO, Lemmon MA, Ferguson KM. Dimerization of Tie2 mediated by its membrane-proximal fniii domains. Proc Natl Acad Sci USA. 2017:114:4382-7

45. Davis S, Papadopoulos N, Aldrich TH, et al. Angiopoietins have distinct modular domains essential for receptor binding, dimerization and superclustering. Nat Struct Biol. 2003;10:38-44.

46. Li Z, Huang $\mathrm{H}$, Boland $\mathrm{P}$, et al. Embryonic stem cell tumor model reveals role of vascular endothelial receptor tyrosine phosphatase in regulating Tie2 pathway in tumor angiogenesis. Proc Natl Acad Sci USA. 2009;106:22399-404.

47. Winderlich M, Keller L, Cagna G, et al. Ve-Ptp controls blood vessel development by balancing Tie-2 activity. J Cell Biol. 2009;185:657-71.

48. Frye M, Dierkes M, Kuppers V, et al. Interfering with Ve-Ptp stabilizes endothelial junctions in vivo via Tie-2 in the absence of ve-cadherin. J Exp Med. 2015;212:2267-87

49. Papapetropoulos A, Fulton D, Mahboubi K, et al. Angiopoietin-1 inhibits endothelial cell apoptosis via the Akt/survivin pathway. J Biol Chem. 2000;275:9102-5.

50. Kontos CD, Cha EH, York JD, Peters KG. The endothelial receptor tyrosine kinase Tie1 activates phosphatidylinositol 3-kinase and akt to inhibit apoptosis. Mol Cell Biol. 2002;22:1704-13.

51. Daly C, Wong $\mathrm{V}$, Burova E, et al. Angiopoietin-1 modulates endothelial cell function and gene expression via the transcription factor Fkhr (Foxo1). Genes Dev. 2004;18:1060-71.
52. Kanda S, Miyata Y, Mochizuki Y, Matsuyama T, Kanetake H. Angiopoietin 1 is mitogenic for cultured endothelial cells. Cancer Res. 2005:65:6820-7.

53. Koblizek TI, Weiss C, Yancopoulos GD, Deutsch U, Risau W. Angiopoietin-1 induces sprouting angiogenesis in vitro. Curr Biol. 1998;8:529-32.

54. Augustin HG, Koh GY, Thurston G, Alitalo K. Control of vascular morphogenesis and homeostasis through the angiopoietin-Tie system. Nat Rev Mol Cell Biol. 2009;10:165-77.

55. Hughes DP, Marron MB, Brindle NP. the antiinflammatory endothelial tyrosine kinase Tie2 interacts with a novel nuclear factor-kappab inhibitor abin-2. Circ Res. 2003;92:630-6.

56. Ziegler T, Horstkotte J, Schwab C, et al. Angiopoietin 2 mediates microvascular and hemodynamic alterations in sepsis. J Clin Invest. 2013.

57. Menden H, Welak S, Cossette S, Ramchandran R, Sampath V. Lipopolysaccharide (Lps)-mediated angiopoietin-2-dependent autocrine angiogenesis is regulated by nadph oxidase 2 (Nox2) in human pulmonary microvascular endothelial cells. J Biol Chem. 2015;290:5449-61.

58. Sato TN, Tozawa Y, Deutsch U, et al. Distinct roles of the receptor tyrosine kinases Tie-1 and Tie-2 in blood vessel formation. Nature. 1995:376:70-4.

59. Dumont DJ, Gradwohl G, Fong GH, et al. Dominant-negative and targeted null mutations in the endothelial receptor tyrosine kinase, tek, reveal a critical role in vasculogenesis of the embryo. Genes Dev. 1994;8:1897-909.

60. Suri C, Jones PF, Patan S, et al. Requisite role of angiopoietin-1, a ligand for the Tie2 receptor, during embryonic angiogenesis. Cell. 1996:87:1171-80.

61. Suri C, McClain J, Thurston G, et al. Increased vascularization in mice overexpressing angiopoietin-1. Science. 1998;282:468-71.

62. Thurston G, Rudge JS, loffe E, et al. Angiopoietin-1 protects the adult vasculature against plasma leakage. Nat Med. 2000;6:460-3.

63. Gale NW, Thurston G, Hackett SF, et al. Angiopoietin-2 is required for postnatal angiogenesis and lymphatic patterning, and only the latter role is rescued by angiopoietin-1. Dev Cell. 2002;3:411-23.

64. Ma L, Brelen ME, Tsujikawa M, et al. Identification of Angpt2 as a new gene for neovascular age-related macular degeneration and polypoidal choroidal vasculopathy in the Chinese and Japanese populations. Invest Ophthalmol Vis Sci. 2017;58:1076-83.

65. Ng DS, Yip YW, Bakthavatsalam M, et al. Elevated angiopoietin 2 in aqueous of patients with neovascular age related macular degeneration correlates with disease severity at presentation. Sci Rep. 2017:7:45081.

66. Hera R, Keramidas M, Peoc'h M, Mouillon M, Romanet JP, Feige JJ. Expression of Vegf and angiopoietins in subfoveal membranes from patients with age-related macular degeneration. Am J Ophthalmol. 2005:139:589-96.

67. Otani A, Takagi H, Oh H, Koyama S, Matsumura M, Honda Y. Expressions of angiopoietins and Tie2 in human choroidal neovascular membranes. Invest Ophthalmol Vis Sci. 1999;40:1912-20.

68. Huber M, Wachtlin J. Vitreous levels of proteins implicated in angiogenesis are modulated in patients with retinal or choroidal neovascularization. Ophthalmologica. 2012;228:188-93.

69. Tuuminen R, Loukovaara S. Increased intravitreal angiopoietin-2 levels in patients with retinal vein occlusion. Acta Ophthalmol. 2014;92:e164-5.

70. Lee J, Park DY, Park DY, et al. Angiopoietin-1 suppresses choroidal neovascularization and vascular leakage. Invest Ophthalmol Vis Sci. 2014:55:2191-9.

71. Peters $S$, Cree IA, Alexander R, et al. Angiopoietin modulation of vascular endothelial growth factor: effects on retinal endothelial cell permeability. Cytokine. 2007;40:144-50.

72. Oh H, Takagi $\mathrm{H}$, Suzuma K, Otani A, Matsumura M, Honda Y. Hypoxia and vascular endothelial growth factor selectively up-regulate angiopoietin-2 in bovine microvascular endothelial cells. J Biol Chem. 1999;274:15732-9.

73. Ohashi $\mathrm{H}$, Takagi H, Koyama S, et al. Alterations in expression of angiopoietins and the Tie-2 receptor in the retina of streptozotocin induced diabetic rats. Mol Vis. 2004;10:608-17.

74. Park SW, Yun JH, Kim JH, Kim KW, Cho CH, Kim JH. Angiopoietin 2 induces pericyte apoptosis via Alpha3beta1 integrin signaling in diabetic retinopathy. Diabetes. 2014;63:3057-68. 
75. Lee SG, Lee CG, Yun IH, Hur DY, Yang JW, Kim HW. Effect of lipoic acid on expression of angiogenic factors in diabetic rat retina. Clin Exp Ophthalmol. 2012;40:e47-57.

76. Watanabe D, Suzuma K, Suzuma I, et al. Vitreous levels of angiopoietin 2 and vascular endothelial growth factor in patients with proliferative diabetic retinopathy. Am J Ophthalmol. 2005;139:476-81.

77. Loukovaara S, Robciuc A, Holopainen JM, et al. Ang-2 upregulation correlates with increased levels of Mmp-9, Vegf, Epo and Tgfbeta1 in diabetic eyes undergoing vitrectomy. Acta Ophthalmol. 2013;91:531-9.

78. Khalaf N, Helmy H, Labib H, Fahmy I, El Hamid MA, Moemen L. Role of angiopoietins and Tie-2 in diabetic retinopathy. Elect Phys. 2017:9:5031-5.

79. Liu XW, Ma T, Liu W, et al. Sustained increase in angiopoietin-2, heparinbinding protein, and procalcitonin is associated with severe sepsis. Crit Care. 2018;45:14-9.

80. Lymperopoulou K, Velissaris D, Kotsaki A, et al. Angiopoietin-2 associations with the underlying infection and sepsis severity. Cytokine. 2015;73:163-8.

81. Tee JK, Setyawati MI, Peng F, Leong DT, Ho HK. Angiopoietin-1 accelerates restoration of endothelial cell barrier integrity from nanoparticleinduced leakiness. Nanotoxicology. 2019;13:682-700.

82. Mirando AC, Shen J, Silva RLE, et al. A collagen IV-derived peptide disrupts alpha5beta1 integrin and potentiates Ang2/Tie2 signaling. JCl Insight 2019:4.

83. Dekker NAM, van Meurs M, van Leeuwen ALI, et al. Vasculotide, an angiopoietin-1 mimetic, reduces pulmonary vascular leakage and preserves microcirculatory perfusion during cardiopulmonary bypass in rats. $\mathrm{Br} J$ Anaesth. 2018;121:1041-51.

84. Gurnik S, Devraj K, Macas J, et al. Angiopoietin-2-induced bloodbrain barrier compromise and increased stroke size are rescued by Ve-Ptp-dependent restoration of Tie2 signaling. Acta Neuropathol. 2016;131:753-73.

85. Rubig E, Stypmann J, Van Slyke P, et al. The synthetic Tie2 agonist peptide vasculotide protects renal vascular barrier function in experimental acute kidney injury. Sci Rep. 2016;6:22111.

86. Wang Y, Wang VM, Chan CC. The role of anti-inflammatory agents in age-related macular degeneration (Amd) treatment. Eye (Lond) 2011:25:127-39.

87. Doganay S, Evereklioglu C, Er H, et al. Comparison of serum No, TnfAlpha, II-1 beta, Sil-2r, II-6 and II-8 levels with grades of retinopathy in patients with diabetes mellitus. Eye (Lond). 2002;16:163-70.

88. Deobhakta A, Chang LK. Inflammation in retinal vein occlusion. Int J Inflam. 2013;2013:438412.

89. Scholz A, Plate KH, Reiss Y. Angiopoietin-2: a multifaceted cytokine that functions in both angiogenesis and inflammation. Ann N Y Acad Sci. 2015:1347:45-51.

90. Gavard J, Patel V, Gutkind JS. Angiopoietin-1 prevents vegf-induced endothelial permeability by sequestering src through Mdia. Dev Cell. 2008;14:25-36.

91. Gavard J, Gutkind JS. Vegf controls endothelial-cell permeability by promoting the beta-arrestin-dependent endocytosis of Ve-cadherin. Nat Cell Biol. 2006;8:1223-34.

92. Silva RLE, Kanan Y, Mirando AC, et al. Tyrosine kinase blocking collagen iv-derived peptide suppresses ocular neovascularization and vascular leakage. Sci Transl Med. 2017;9.

93. Mirando AC, Silva RLE, Chu Z, Campochiaro P, Popel AS, Pandey NB. Supression of ocular inflammation by a Tie2 activating peptide (in Press).

94. Senger DR, Davis GE. Angiogenesis. Cold Spring Harbor Persp Biol. 2011;3:a005090.

95. O'Reilly MS, Boehm T, Shing Y, et al. Endostatin: an endogenous inhibitor of angiogenesis and tumor growth. Cell. 1997;88:277-85.

96. Maeshima Y, Colorado PC, Torre A, et al. Distinct antitumor properties of a type Iv collagen domain derived from basement membrane. J Biol Chem. 2000;275:21340-8.

97. Karagiannis ED, Popel AS. A systematic methodology for proteomewide identification of peptides inhibiting the proliferation and migration of endothelial cells. Proc Natl Acad Sci USA. 2008;105:13775-80.

98. Francis SE, Goh KL, Hodivala-Dilke K, et al. Central roles of alpha5beta1 integrin and fibronectin in vascular development in mouse embryos and embryoid bodies. Arterioscler Thromb Vasc Biol. 2002;22:927-33.
99. Hynes RO. Cell-matrix adhesion in vascular development. J Thromb Haemost. 2007;5(Suppl 1):32-40.

100. Umeda N, Kachi S, Akiyama H, et al. Suppression and regression of choroidal neovascularization by systemic administration of an alpha5beta1 integrin antagonist. Mol Pharmacol. 2006;69:1820-8.

101. Soldi R, Mitola S, Strasly M, Defilippi P, Tarone G, Bussolino F. Role of alphavbeta3 integrin in the activation of vascular endothelial growth factor receptor-2. EMBO J. 1999;18:882-92.

102. Regula JT, Lundh von Leithner P, Foxton $R$, et al. Targeting key angiogenic pathways with a bispecific crossmab optimized for neovascular eye diseases. EMBO Mol Med. 2016;8:1265-88.

103. Gahn GM, Khanani AM. New therapies of neovascular amd beyond anti-vegf injections. Vision (Basel). 2018;2.

104. Helzner J. Faricimab shows potential for 16-week dosing: initial data presented from genentech's stairway study. Retinal Phys. 2018.

105. Khanani AM. Simulataneous Inhibition of Ang-2 and Vegf with faricimab in neovascular amd: stairway phase 2 results. American academy of ophthalmology retina subspecialty day. San Franciso, CA2019.

106. Sahni J, Patel SS, Dugel PU, et al. Simultaneous inhibition of angiopoietin-2 and vascular endothelial growth factor-a with faricimab in diabetic macular edema: boulevard phase 2 randomized trial. Ophthalmology. 2019;126:1155-70

107. Clinicaltrials. Gov. A study to evaluate the efficacy and safety of faricimab in participants with neovascular age-related macular degeneration (Tenaya). https://clinicaltrials.gov/ct2/show/NCT0382328 7?term =faricimab\&draw=2\&rank=1.). Accessed 25 Nov 2019.

108. Clinicaltrials. Gov. A study to evaluate the efficacy and safety of faricimab in participants with neovascular age-related macular degeneration (Lucerne). https://clinicaltrials.gov/ct2/show/NCT0382330 0?term=faricimab\&draw=2\&rank=2.). Accessed 25 Nov 2019.

109. Clinicaltrials.Gov. A study to evaluate the efficacy and safety of faricimab (Ro6867461) in participants with diabetic macular edema (Rhine). (Accessed April 4, 2020, at a study to evaluate the efficacy and safety of Faricimab (RO6867461) in participants with diabetic macular edema (RHINE).).

110. Clinicaltrials. Gov. A study to evaluate the efficacy and safety of faricimab (Ro6867461) in participants with diabetic macular edema (Yosemite). https://clinicaltrials.gov/ct2/show/NCT0362258 0 ?term $=$ faricimab\&draw $=2 \&$ rank=3.). Accessed 4 April 2020.

111. Shen J, Frye M, Lee BL, et al. Targeting Ve-Ptp activates Tie2 and stabilizes the ocular vasculature. J Clin Invest. 2014;124:4564-76.

112. Campochiaro PA, Sophie R, Tolentino M, et al. Treatment of diabetic macular edema with an inhibitor of vascular endothelial-protein tyrosine phosphatase that activates Tie2. Ophthalmology. 2015;122:545-54.

113. Campochiaro PA, Khanani A, Singer M, et al. Enhanced benefit in diabetic macular edema from Akb-9778 Tie2 activation combined with vascular endothelial growth factor suppression. Ophthalmology. 2016;123:1722-30.

114. Aerpio Press Release (February 10, 2016). Aerpio therapeutics announces presentation of positive results of Akb-9778 in patients with diabetic retinopathy from time-2 phase 2a study. https://www.busin esswire.com/news/home/20160210006109/en/Aerpio-Therapeuti cs-Announces-Presentation-Positive-Results-AKB-9778. Accessed 4 Apr 2020

115. Aerpio Corporate Presentation. 2019. http://ir.aerpio.com/static-files /7ec12cbe-9bfd-41e4-98fb-569070cd1bf1. Accessed 7 Apr 2020.

116. Clinicaltrials. Gov. Open label study to assess the efficacy and safety of Akb-9778 in subjects with macular edema due to Rvo. https://clinicaltr ials.gov/ct2/show/NCT02387788?term =akb-9778\&draw $=2 \&$ rank=2. Accessed 4 Apr2020.

117. Aerpio. Arp-1536. https://aerpio.com/pipeline/arp-1536/. Accessed 4 Apr 2020.

118. Brown DM. Phase 1 Study of combination therapy with nesvacumab and aflibercept for neovascular amd and diabetic macular edema. In: American academy of ophthalmology annual meeting. Chicago, IL2016.

119. Clinicaltrials. Gov. Anti-angiopoeitin 2 plus anti-vascular endothelial growth factor as a therapy for neovascular age related macular degeneration: evaluation of a fixed combination intravitreal injection (Onyx). https://clinicaltrials.gov/ct2/show/NCT02713204?term=NCT0271320 4\&draw=2\&rank=1.). Accessed 4 Apr 2020. 
120. Clinicaltrials. Gov. Anti-vascular endothelial growth factor plus antiangiopoietin 2 in fixed combination therapy: evaluation for the treatment of diabetic macular edema (Ruby). https://clinicaltrials.gov/ct2/ show/NCT02712008?term $=$ nesvacumab\&draw $=2 \&$ rank $=3$.). Accessed 4 Apr 2020.

121. Regeneron Press Release (November 27, 2017). Regeneron Provides Update on Eylea ${ }^{\circledR}$ (Aflibercept) Injection and Nesvacumab (Ang2 Antibody) Combination Program. https://investor.regeneron.com/ news-releases/news-release-details/regeneron-provides-update-eylea r-aflibercept-injection-and. Accessed 4 Apr 2020.

\section{Publisher's Note}

Springer Nature remains neutral with regard to jurisdictional claims in published maps and institutional affiliations.
Ready to submit your research? Choose BMC and benefit from:

- fast, convenient online submission

- thorough peer review by experienced researchers in your field

- rapid publication on acceptance

- support for research data, including large and complex data types

- gold Open Access which fosters wider collaboration and increased citations

- maximum visibility for your research: over $100 \mathrm{M}$ website views per year

At BMC, research is always in progress.

Learn more biomedcentral.com/submissions 